\title{
Title
}

\section{Role of $\alpha$-Synuclein in Cell Biology: A Hypothesis}

\section{Author}

Renu Minda

\begin{abstract}
I wish to suggest a hypothesis regarding the physiological function of $\alpha$-synuclein, a protein implicated in Parkinson disease, whose cell biology is still debated. The essence is the concept of Carnot cycle of refrigeration in which membrane fluidity depends on the heat produced by mitochondria. The hypothesis has the potential to explain the diverse phenotypes attributed to $\alpha$-synuclein under both physiological and pathological conditions.
\end{abstract}

\section{Keywords}

Parkinson disease, $\alpha$-synuclein, thermodynamics, Carnot cycle, and mitochondria

\section{Contact details}

E-mail: $\underline{\text { mindarenu@gmail.com }}$

\section{Affiliation}

Ex. Research Fellow, Dept. of Biosciences,

Sri Sathya Sai Institute of Higher Learning,

Puttaparthi, Andhra Pradesh, India.

ORCID id: 0000-0002-2359-9146 


\section{Main Text}

$\alpha$-Synuclein, an intrinsically disordered protein, enriched in pre-synaptic nerve boutons of the central nervous system of vertebrates, became the focus of intense pathology oriented research after being identified in 1997 as the first gene (SNCA) causing a monogenic form of Parkinson Disease $(P D)^{1}$. Despite decades of extensive investigations, the neurodegeneration caused by $\alpha$-synuclein is poorly understood, and its cell biology also remains obscure ${ }^{2}$. In the present study an attempt has been made to reconcile some of the diverse findings associated with $\alpha$-synuclein under both physiological and pathological conditions, using a thermodynamic approach. The hypothesis assumes that $\alpha$-synuclein functions as a refrigerant of Carnot cycle in a sub-cellular milieu.

Cells of living organisms are steady-state non-equilibrium systems. However, the application of the principles of closed system thermodynamics is considered valid as an approximation in biological processes ${ }^{3}$. The hypothesis assumes the tenets of equilibrium thermodynamics.

Biological cells function at essentially constant temperature, nonetheless, temperature gradients do exist as a result of heterogeneity of energy flow in metabolism. Mitochondria, nucleus, and the endoplasmic reticulum generate heat ${ }^{4-6}$. A steep temperature gradient exists between mitochondria and the cell membrane ${ }^{7-9}$. A gradient (for example, in temperature, pressure, or chemical concentration) can establish work cycles locally. My conjecture is that $\alpha$-synuclein taps the temperature gradient between mitochondria and the membrane and performs its biological function - decreasing membrane fluidity.

Heat naturally flows in direction of decreasing temperature. For the reverse, that is, for heat to flow from a low-temperature reservoir to a high-temperature reservoir requires 
work which is done by a compressor in a refrigerator using electrical energy. In the proposed model the work done by the compressor in a refrigerator is substituted by thermal energy produced by mitochondria and the working substance is $\alpha$-synuclein operating in a cycle in both its monomeric and its fibrillar form. The membrane in liquid-disordered phase, forms the low temperature reservoir and the cytosol functions as the heat sink, the high temperature reservoir of the Carnot cycle. During each cycle, $\alpha$-synuclein monomers, working as a refrigerant, transfers heat from the membrane to the cytosol. Consequently, $\alpha$-synuclein monomers are converted into fibrils and the membrane undergoes a phase transition from liquid-disordered to solid gel-state. Membrane phase transition is an isothermal process comparable to formation of ice from liquid water at its freezing point. It takes place without change of temperature though heat transfer occurs. Mitochondria serve to provide thermal energy necessary for the continuous operation of the $\alpha$-synuclein associated refrigeration; by creating a temperature gradient and by converting $\alpha$-synuclein fibrils back to monomers. Consistent with this assumption, in in vitro studies $\alpha$-synuclein fibrils are noted to be unusually heat labile ${ }^{10-11}$. To maintain functional stoichiometry, the dynamic steady state level of $\alpha$-synuclein at the nerve terminus is coupled to efflux of non-functional fibrils through scavenging activities and influx of freshly synthesised monomers (not shown in the figure).

Evidence for different steps of the $\alpha$-synuclein associated Carnot cycle can be identified from the published literature referred here. Essential feature of the model is reversible interaction of $\alpha$-synuclein with the membrane ${ }^{12}$. On association with lipids in membrane $\alpha$-synuclein takes up an $\alpha$-helical conformation, this induces lipids to undergo a phase transition from liquid-disordered to solid gel state ${ }^{13-14}$, thereby releasing the internal energy of lipids as heat into the cytosol, which in turn induces the NAC (non-AB-amyloid 
component) domain of $\alpha$-synuclein to undergo a conformation change from alpha helix to beta-sheet ${ }^{15}$. This acts as a nucleus to recruit free monomeric $\alpha$-synuclein from the cytosol to take up beta-sheet conformation and form fibrils on the surface of the membrane. This is an ordered, low entropy state, following release of energy necessary for dislodging fibrils from the membrane. Fibrils are a consequence not only of the phase transition of the membrane but also of the energy that is degraded. Monomers, before interaction with the membrane, are disordered and have a high internal energy. Fibrils, formed after interaction with the membrane, are ordered and have a low internal energy. Order in $\alpha$-synuclein fibrils and in the membrane is at the expense of rise in entropy of the cytosol.

The model proposes a regulation of membrane fluidity by working of the Carnot cycle.

Alteration in membrane fluidity can explain the following phenotypes attributed to $\alpha$ synuclein's presence or absence under physiological conditions:

1. $\alpha$-Synuclein mRNA levels change during song acquisition in zebra finches ${ }^{16} \Rightarrow$ neuronal plasticity involves structural changes in membrane which in turn is regulated by membrane fluidity.

2. $\alpha$-Synuclein knockout mice exhibit a reduction in the reserve pool of synaptic vesicles in hippocampal synapses compared to wild type controls ${ }^{17} \Rightarrow$ loss of $\alpha$-synuclein causes the membrane to persist in fluid state which leads to vesicle clustering ${ }^{18-20}$.

3. Dopamine level in the striatum is reduced in $\alpha$-synuclein null mice compared to wild type controls ${ }^{21} \Rightarrow$ loss of $\alpha$-synuclein causes the membrane to remain in fluid state thereby increasing membrane permeability which leads to a storage defect of synaptic vesicles ${ }^{22}$. 
4. Nerve conduction velocity in aged synuclein triple knockout mice is reduced compared to age-matched wild type controls ${ }^{23} \Rightarrow$ loss of $\alpha$-synuclein causes the membrane to persist in fluid state that correlates with a decrease in membrane capacitance thereby decreasing nerve conduction velocity ${ }^{24}$.

Perturbation in the $\alpha$-synuclein associated Carnot cycle can explain the following phenotypes ascribed to $\alpha$-synuclein under pathological conditions:

1. Increased copy number of $\alpha$-synuclein gene $(S N C A)$ cause early onset $P^{25} \Rightarrow$ for the steady-state operation of the Carnot cycle appropriate concentration of $\alpha$-synuclein protein at the nerve terminus has to be maintained - an increased flux of protein may lead to increased membrane frigidity and/or increased accumulation of $\alpha$-synuclein fibrils and both conditions can be toxic.

2. Missense mutations in $\alpha$-synuclein that cause PD can be broadly divided into two categories: those that increase the rate of self-aggregation (A53T, H50Q, and E46K), and those that reduce the rate of self-aggregation, and also impairs the ability of $\alpha$ synuclein to bind to lipids and brain vesicles (A30P, G51D, and A53E) ${ }^{26} \Rightarrow$ for the steady-state operation of the Carnot cycle, equilibrium between $\alpha$-synuclein monomers and fibrils in the cytosol and between free monomeric, unbound $\alpha$ synuclein and membrane bound form must be maintained. A disturbance in this equilibrium may cause toxicity.

3. Mitochondrial dysfunction is related to $\alpha$-synuclein accumulation in $\mathrm{PD}^{27} \Rightarrow$ If mitochondria are non-functional $\alpha$-synuclein fibrils will not be converted back to monomers and consequently fibrils will accumulate. 
It is apparent that the hypothesis has wider implication. Any factor that disturbs the steady-state functioning of the $\alpha$-synuclein associated Carnot cycle, other than that due to quantitative and structural change in $\alpha$-synuclein protein, may cause neurodegeneration, as may be the case in the more common form of PD called "idiopathic". The specific links to the cellular metabolic web with which the hypothesized cycle interacts may give intelligible clues linking the several known risk factors annotated in genome wide association analysis ${ }^{25}$.

The novel feature of the hypothesis is that mitochondrial heat at the nerve terminus is not wasted: it is used to disaggregate $\alpha$-synuclein fibrils and to create a temperature gradient for doing work in thermodynamic sense in a Carnot cycle. One test this hypothesis can be subjected to is by monitoring the $\alpha$-synuclein fibril dissociation rate in the presence of uncoupled mitochondria.

\section{Acknowledgement}

I am grateful to Dr. Venketesh Sivaramakrishnan for critically reading the manuscript and for inducting me to the exciting field of Parkinson disease.

\section{Competing Interest Declaration}

I declare no competing interest. 


\section{References}

1. Polymeropoulos, M. H. et al. Mutation in the alpha-synuclein gene identified in families with Parkinson's disease. Science 276, 2045-2047 (1997).

2. Burré, J., Sharma, M. \& Südhof, T. C. Cell Biology and Pathophysiology of $\alpha$-Synuclein. Cold Spring Harb. Perspect. Med. 8, (2018)

3. Harold, F. The Vital Force: A Study of Bioenergetics Ch. 1 (W. H. Freeman and Co., New York, 1986).

4. Andrews, Z., Diano, S. \& Horvath, T. Mitochondrial uncoupling proteins in the cns: in support of function and survival. Nat. Rev. Neurosci. 6, 829-840 (2005).

5. Okabe, K. et al. Intracellular temperature mapping with a fluorescent polymeric thermometer and fluorescence lifetime imaging microscopy. Nat. Commun. 3, 705 (2012).

6. Bal, N. C. et al. Sarcolipin is a newly identified regulator of muscle-based thermogenesis in mammals. Nat. Med. 18, 1575-1579 (2012).

7. Rajagopal, M. C. et al. Transient heat release during induced mitochondrial proton uncoupling. Commun. Biol. 2, 279 (2019).

8. Chre'tien, D. et al. Mitochondria are physiologically maintained at close to $50{ }^{\circ} \mathrm{C}$. PLoS Biol. 16, (2018).

9. Lane, N. Hot mitochondria? PLoS Biol. 16, (2018).

10. van Gils, J. H. M. et al. The hydrophobic effect characterises the thermodynamic signature of amyloid fibril growth. PLoS Comput. Biol. 16, (2020).

11. Skamris, T., Marasini, C., Madsen, K. L., Fodera, V., \& Vestergaard, B. Early Stage Alpha-Synuclein Amyloid Fibrils are Reservoirs of Membrane-Binding Species. Sci. Rep. 9, 1733 (2019). 
12. Jao, C. C., Hegde, B. G., Chen, J., Haworth, I. S., \& Langen, R. Structure of membranebound alpha-synuclein from site-directed spin labeling and computational refinement. Proc. Natl. Acad. Sci. U S A. 105, 19666-19671 (2008).

13. Kamp, F. \& Beyer, K. Binding of alpha-synuclein affects the lipid packing in bilayers of small vesicles. J. Biol. Chem. 281, 9251-9259 (2006).

14. Nuscher, B. et al. Alpha-synuclein has a high affinity for packing defects in a bilayer membrane: a thermodynamics study. J. Biol. Chem. 279, 21966-21975 (2004).

15. Galvagnion, C. et al. Lipid vesicles trigger $\alpha$-synuclein aggregation by stimulating primary nucleation. Nat. Chem. Biol. 11, 229-234 (2015).

16. George, J. M., Jin, H., Woods, W. S., \& Clayton, D. F. Characterization of a novel protein regulated during the critical period for song learning in the zebra finch. Neuron 15, 361-372 (1995).

17. Cabin, D. E. et al. Synaptic vesicle depletion correlates with attenuated synaptic responses to prolonged repetitive stimulation in mice lacking alpha-synuclein. J. Neurosci. 22, 8797-8807 (2002).

18. Zaltieri, M. et al. $\alpha$-synuclein and synapsin III cooperatively regulate synaptic function in dopamine neurons. J. Cell Sci. 128, 2231-2243 (2015).

19. Lim, L. \& Wenk, M. R. Neuronal Membrane Lipids - Their Role in the Synaptic Vesicle Cycle. In: Handbook of Neurochemistry and Molecular Neurobiology. (eds. Lajtha, A., Tettamanti, G., \& Goracci, G.) 223-238 (Springer, Boston, MA, 2009).

20. Lentz, B. R., Carpenter, T. J., \& Alford, D. R. Spontaneous fusion of phosphatidylcholine small unilamellar vesicles in the fluid phase. Biochemistry 26, 5389-5397 (1987). 
21. Abeliovich, A. et al. Mice lacking alpha-synuclein display functional deficits in the nigrostriatal dopamine system. Neuron 25, 239-252 (2000).

22. Li, Z. et al. The ratio of phosphatidylcholine to phosphatidylethanolamine influences membrane integrity and steatohepatitis. Cell Metab. 3, 321-331 (2006).

23. Greten-Harrison, B. et al. $\alpha \beta \gamma$-Synuclein triple knockout mice reveal age-dependent neuronal dysfunction. Proc. Natl. Acad. Sci. U S A. 107, 19573-19578 (2010).

24. Heo, P., \& Pincet, F. Freezing and piercing of in vitro asymmetric plasma membrane by $\alpha$-synuclein. Commun. Biol. 3, 148 (2020).

25. Lill, C. M. \& Klein, C. The Neurogenetics of Parkinson's Disease and Putative Links to Other Neurodegenerative Disorders. In: Parkinson's Disease: Molecular Mechanisms Underlying Pathology. (ed: Verstreken, P.) 1-40 (Academic Press, Cambridge, 2017).

26. Tofaris, G. K., Goedert, M. \& Spillantini, M. G. The Transcellular Propagation and Intracellular Trafficking of $\alpha$-Synuclein. Cold Spring Harb. Perspect. Med. 7, (2017).

27. Aerts, L. \& Morais, V. A. Electron Transport Chain. In: Parkinson's Disease: Molecular Mechanisms Underlying Pathology. (ed: Verstreken, P.) 41-75 (Academic Press, Cambridge, 2017. 